\title{
MAXIMAL EXTENSIONS OF FIRST-COUNTABLE SPACES
}

\author{
TOSHIJI TERADA AND JUN TERASAWA
}

\begin{abstract}
A first-countable space is called maximal if it is not contained as a dense subspace in a first-countable space properly. The following are shown; (1) every locally compact, first-countable space is a dense subspace of a maximal space, (2) every metrizable space is a dense subspace of a maximal space, and (3) there is a first-countable space which is not a dense subspace of any maximal space.
\end{abstract}

All spaces in this paper are Tychonoff unless otherwise specified.

A space which contains a space $X$ as a dense subspace is called an extension of $X$. Let us call a first-countable space maximal, or, more precisely, maximal with respect to first-countability, if it has no proper, first-countable extension. According to [S, Theorem 2.9], a first-countable space is maximal if and only if it is pseudocompact. (Note that our maximal spaces are identical to Stephenson's first countableand completely regular-closed spaces.) Hence, if a first-countable space $X$ has first-countable compactification $Y$, then $Y$ is a maximal extension of $X$. On the other hand, even if $X$ does not have a first-countable compactification, it can still have a maximal extension.

Here, we are concerned with two questions. Namely, which first-countable spaces have maximal extensions, and whether all first-countable spaces have maximal extensions.

In $\$ 1$ and 2, we shall answer the first question by showing that every locally compact, first-countable space and every metrizable space have maximal extensions. $\$ 3$ will provide a negative answer to the second question.

\section{Locally compact, first-countable spaces.}

1.1. We shall begin with

Proposition. For a first-countable space $X$, the following conditions are equivalent:

(1) $X$ has a maximal extension,

(2) there are a maximal disjoint collection $\mathscr{Z}=\left\{Z_{\alpha} \mid \alpha \in A\right\}$ of zero-sets of $\beta X$ and $a$ family $\mathscr{F}=\left\{f_{\alpha} \mid \alpha \in A\right\}$ of real-valued continuous functions on $\beta X$ such that

(a) $\{x\} \in \mathscr{Z}$ for each $x \in X$,

(b) $f_{\alpha}^{-1}(0)=Z_{\alpha}$ for each $\alpha \in A$, and

(c) $f_{\alpha} \mid Z_{\beta}$ is constant for any $\alpha \in A$ and $\beta \in A$.

Received by the editors February 19, 1981 and, in revised form, August 11, 1981.

1980 Mathematics Subject Classification. Primary 54D25, 54C25.

Key words and phrases. First-countable, pseudocompact, maximal extension, locally compact, metrizable, Čech-complete. 
Proof. (1) $\rightarrow$ (2) Let $Y$ be a maximal extension of $X$. Then $Y$ is first-countable and pseudocompact. Since $\beta Y$ is a compactification of $X$, we have a continuous surjection $h: \beta X \rightarrow \beta Y$ which is identical on $X$. For each $y \in Y$, there is a real-valued continuous function $f_{y}$ on $\beta Y$ such that $f_{y^{\prime}}^{-1}(0)=\{y\}$. Let $\mathscr{Z}=\left\{h^{-1}(y) \mid y\right.$ $\in Y\}$ and $\mathscr{F}=\left\{f_{y} \circ h \mid y \in Y\right\}$. Then $\mathscr{Z}$ and $\mathscr{F}$ satisfy the conditions (a) to (c). To see that $\mathscr{Z}$ is a maximal disjoint collection of zero-sets of $\beta X$ it suffices to observe that $h^{-1}(Y)$ is pseudocompact. This is shown by noting that the restriction $h \mid h^{-1}(Y)$ is perfect and irreducible, and that the preimage of a pseudocompact space under a perfect and irreducible map is always pseudocompact.

(2) $\rightarrow$ (1) Let $W=\cup\left\{Z_{\alpha} \mid \alpha \in A\right\}$ and $Y$ be a quotient space of $W$ obtained by collapsing each $Z_{\alpha}$ to a point. Obviously, $Y$ is a first-countable, Tychonoff extension of $X$. The maximality of $\mathscr{Z}$ implies that $W$ is pseudocompact, hence so is $Y$. Therefore $Y$ is a maximal extension of $X$.

1.2. THEOREM. Every locally compact, first-countable space has a maximal extension.

Proof. Let $X$ be locally compact and first-countable. At first consider a cozero-set $C$ of $X^{*}=\beta X-X$ and a zero-set $Z$ of $\beta X$ such that $C \subset Z \subset X^{*}$. Then there is a continuous function $g: X^{*} \rightarrow \mathbf{R}$ such that $C=g^{-1}(\mathbf{R}-\{0\})$. (Here $\mathbf{R}$ denotes the space of reals.) Since $X$ is locally compact, $X^{*}$ is a closed subset of a compact space $\beta X$ and $g$ has a continuous extension $h: \beta X \rightarrow \mathbf{R}$. Take any $r \neq 0$. We have that $g^{-1}(r)$ is a $G_{\delta}$-set of $X^{*}$, that $Z$ is a $G_{\delta}$-set of $\beta X$ and that $g^{-1}(r) \subset Z \subset X^{*}$. Hence $g^{-1}(r)$ is a $G_{\delta}$-set of $\beta X$ and is expressed as $g^{-1}(r)=\bigcap\left\{U_{n} \mid n=1,2, \ldots\right\}$ with open sets $U_{n}$ of $\beta X$. For each $n, h^{-1}(r)-U_{n}$ and $X^{*}$ are disjoint closed subsets of $\beta X$. There is a continuous map $k_{n}: \beta X \rightarrow[0,1]$ such that $k_{n}\left(X^{*}\right)=\{0\}$ and $k_{n}\left(h^{-1}(r)-U_{n}\right)=\{1\}$. Define $f=\sum_{n=1}^{\infty}\left(1 / 2^{n}\right) k_{n}+|h-r|$. Then $f$ is a real-valued continuous function on $\beta X$ such that $f\left|X^{*}=\right| g-r \mid$ and $f^{-1}(0)=g^{-1}(r)$.

Next, consider the collection of all cozero-sets $C$ of $X^{*}$ such that $C \subset Z \subset X^{*}$ for some zero-set $Z$ of $\beta X$. Take a maximal disjoint subcollection $\left\{C_{\lambda} \mid \lambda \in \Lambda\right\}$ of this collection. For each $\lambda \in \Lambda$, there is a continuous function $g_{\lambda}: X^{*} \rightarrow \mathbf{R}$ such that $C_{\lambda}=g_{\lambda}^{-1}(\mathbf{R}-\{0\})$. As above, for each $\lambda \in \Lambda$ and $r \neq 0$, there is a continuous function $f_{\lambda, r}: \beta X \rightarrow \mathbf{R}$ such that $f_{\lambda, r}\left|X^{*}=\right| g_{\lambda}-r \mid$ and $f_{\lambda, r}^{-1}(0)=g_{\lambda}^{-1}(r)$. Hence $Z_{\lambda, r}=g_{\lambda}^{-1}(r)$ is a zero-set of $\beta X$ and $f_{\lambda, r} \mid Z_{\lambda^{\prime}, r^{\prime}}$ is constant for any $(\lambda, r)$ and $\left(\lambda^{\prime}, r^{\prime}\right)$.

Moreover, if $Z \subset X^{*}$ is a zero-set of $\beta X$, then int $x^{*} Z \neq \varnothing$ because $X$ is locally compact (see the proof of [W,4.21]). Therefore int ${ }_{X^{*}} Z$, and hence $Z$, contains a cozero-set of $X^{*}$. This means that $\{\{x\} \mid x \in X\} \cup\left\{Z_{\lambda, r} \mid(\lambda, r) \in \Lambda \times(\mathbf{R}-\{0\})\right\}$ forms a maximal disjoint collection of zero-sets of $\beta X$. For each $x \in X$ let $f_{x}$ be a real-valued continuous function on $\beta X$ such that $f_{x}^{-1}(0)=\{x\}$ and $f_{x}\left(X^{*}\right)=\{1\}$. Then it is now easy to check that

$$
\{\{x\} \mid x \in X\} \cup\left\{Z_{\lambda, r} \mid(\lambda, r) \in \Lambda \times(\mathbf{R}-\{0\})\right\}
$$

and

$$
\left\{\left\{f_{x}\right\} \mid x \in X\right\} \cup\left\{f_{\lambda, r} \mid(\lambda, r) \in \Lambda \times(\mathbf{R}-\{0\})\right\}
$$

satisfy condition (2) of Proposition 1.1. 
1.3. A space is called locally pseudocompact if every point has a pseudocompact neighborhood. For any locally pseudocompact space $X$ we can find a locally compact space $Y$ such that $X \subset Y \subset v X$. In 1.2, let us consider cozero-sets of $\beta X-Y$ instead of $X^{*}$ and note that every zero-set of $\beta X$ which meets $Y \subset v X$ meets $X$. Then we get an extension of $X$ as a quotient space of a subspace of $X \cup(\beta X-Y)$. That is,

THEOREM. Every locally pseudocompact, first-countable space has a maximal extension.

1.4. Since $f_{\lambda, r}^{-1}([-r / 2, r / 2]) \subset X \cup C_{\lambda}$, the maximal extension of $X$ in 1.2 is indeed locally compact. That is,

THEOREM. Every locally compact, first-countable space has a locally compact, maximal extension.

\section{Metrizable spaces.}

2.1. Let $X$ be a space and $A$ its dense subspace. If every sequence in $A$ contains a subsequence which converges in $X$, then we call $X$ e-sequentially compact with respect to $A$. It is not difficult to observe that

(1) if $X$ is $e$-sequentially compact with respect to some dense subset, then $X$ is pseudocompact,

(2) if $X$ is $e$-sequentially compact with respect to $A$, and $B \subset A$, then $\mathrm{cl}_{X} B$ is $e$-sequentially compact with respect to $B$, and

(3) if $X_{n}$ is $e$-sequentially compact with respect to $A_{n}$ for each $n=1,2, \ldots$, then so is the product $\prod_{n=1}^{\infty} X_{n}$ with respect to $\prod_{n=1}^{\infty} A_{n}$.

2.2. Let us recall the following. Let $A$ be a set of cardinality $\mathfrak{m} \geqslant \boldsymbol{\aleph}_{0}$. The star-space $S(A)$ is the metric space $\{0\} \cup \cup\{(0,1] \times\{\alpha\} \mid \alpha \in A\}$ in which the metric function $d$ is defined by: $d(0,(t, \alpha))=t, d((t, \alpha),(s, \beta))=|t-s|$ if $\alpha=\beta$, and $=t+s$ if $\alpha \neq \beta$. In [E, Example 4.1.5], the star-space is called the hedgehog space of spininess $\mathrm{m}$. According to [N, Theorem VI.9], any metrizable space of weight $\leqslant \mathrm{m}$ is homeomorphic to a subspace of the product of countably many copies of $S(A)$.

2.3. We shall show

Proposition. For each $A$, there is a first-countable space $X$ which is e-sequentially compact with respect to $S(A)$.

2.4. By virtue of 2.1 and 2.2, Proposition 2.3 immediately implies

THEOREM. Every metrizable space $M$ has a maximal extension which is e-sequentially compact with respect to $M$.

2.5. Proof of Proposition 2.3. Consider $A$ as a discrete space based on the set $A$. Then $A$ can be embedded as a dense subspace in a first-countable pseudocompact space $B$. (This can be shown in many ways. For example, apply Theorem 1.2 in $\S 1$, or see the proof of $[\mathbf{A}, 2.3 .16]$, or take a maximal almost-disjoint collection $R$ of countably infinite subsets of $A$ and topologize $A \cup \Re$ just as the spaces $N \cup \Re$ in [T].) Take the set $X=\{0\} \cup \cup\{(0,1] \times\{\beta\} \mid \beta \in B\}$. We topologize $X$ in the 
following way: The point 0 has the neighborhood basis

$$
\{0\} \cup \cup\{(0,1 / n) \times\{\beta\} \mid \beta \in B\}, \quad n=1,2, \ldots,
$$

and $(t, \beta) \in(0,1] \times\{\beta\}$ has the neighborhood basis

$$
((t-1 / n, t+1 / n) \cap(0,1]) \times U_{n}, \quad n=1,2, \ldots,
$$

where $\left\{U_{n} \mid n=1,2, \ldots\right\}$ is a neighborhood basis at the point $\beta$ in $B$. Now, it is easy to check that $X$ is a first-countable Tychonoff space and is $e$-sequentially compact with respect to $S(A)$.

\section{First-countable spaces without maximal extensions.}

3.1. In [vDP], a first-countable Lindelöf space $\Delta$ is constructed so that all compactifications of $\Delta$ contain $\beta N$. This $\Delta$ has no maximal extension. The authors incidentally, do not know if there exists a maximal space every compactification of which contains $\beta N$.

3.2. $\Delta$ is not Čech-complete. In view of Theorem 1.2 in $\$ 1$, we present a Čech-complete modification $\Delta^{\prime}$ of $\Delta$. Let $N$ be the set of natural numbers and take any almost-disjoint collection $\Re$ of infinite subsets of $N$ such that $|\Re|=c$. (A collection of sets is called almost-disjoint if the intersection of any two of its elements is a finite set.) The collection of all subsets of $N$ has the cardinality $c$ and can be denoted by $\left\{A_{\lambda} \mid \lambda \in \Re\right\}$. Topologize the set $\Delta^{\prime}=\Re \cup N \times N$ in the following way. All points of $N \times N$ are isolated and the neighborhood basis at $\lambda \in R$ consists of sets $\{\lambda\} \cup((\lambda-$ (finite set $\left.)) \times A_{\lambda}\right)$. It can be easily seen that $\Delta^{\prime}$ is 0 -dimensional, Tychonoff and first-countable. To see that $\Delta^{\prime}$ is Čech-complete, let

$$
\mathscr{Q}_{n}=\left\{\{\lambda\} \cup\left((\lambda-\{1,2, \ldots, n\}) \times A_{\lambda}\right) \mid \lambda \in \mathscr{R}\right\}
$$

and

$$
\mathscr{V}_{n}=\mathscr{U}_{n} \cup\left\{\{x\} \mid x \in \Delta^{\prime}-\cup \mathscr{U}_{n}\right\} \text { for } n=1,2, \ldots
$$

Then each $\mathscr{V}_{n}$ is an open cover of $\Delta^{\prime}$ and $\left\{\mathscr{V}_{n} \mid n=1,2, \ldots\right\}$ determines Čechcompleteness of $\Delta^{\prime}$ by [E, Theorem 3.9.2]. Finally, let us show that $\Delta^{\prime}$ has no first-countable, pseudocompact regular extension. We take any first-countable pseudocompact extension $X$ of $\Delta^{\prime}$ and prove that $X$ is not regular. First, decompose $N$ into countably many, disjoint, infinite sets: $N=\cup_{n=1}^{\infty} B_{n}$. Each $\{n\} \times N$ is a clopen (i.e., simultaneously-closed-and-open) set of $\Delta^{\prime}$, hence so is $\{n\} \times B_{n}$. Since $X$ is pseudocompact, $\{n\} \times B_{n}$ cannot be a closed set of $X$. Pick an accumulation point $x_{n}$ of $\{n\} \times B_{n}$ in $X$ for each $n$. It follows from the first-countability of $X$ that there is an infinite subset $C_{n} \subset B_{n}$ such that $\{n\} \times C_{n}$, as a sequence, converges to $x_{n}$. Decompose $C_{n}$ into two disjoint, infinite sets: $C_{n}=D_{n} \cup D_{n}^{\prime}$. There is a $\lambda \in \Re$ such that $A_{\lambda}=\cup_{n=1}^{\infty} D_{n}$. Obviously $A_{\lambda} \cap \cup_{n=1}^{\infty} D_{n}^{\prime}=\varnothing$. Now assume that $X$ is regular. Then open sets $U_{n}, n=1,2, \ldots$, of $X$, each of which satisfies $\{\lambda\} \cup((\lambda-$ $\left.\{1,2, \ldots, n\}) \times A_{\lambda}\right)=U_{n} \cap \Delta^{\prime}$, form a neighborhood basis at $\lambda$ in $X$. Again, by the regularity of $X, \operatorname{cl}_{X} U_{n} \subset U_{1}$ for some $n$. Take any $m \in \lambda-\{1,2, \ldots, n\}$. Then $\{m\} \times D_{m} \subset U_{n}$. On the other hand, $X-U_{1} \supset \Delta^{\prime}-U_{n} \supset\{m\} \times D_{m}^{\prime}$. Here is a contradiction because both $\{m\} \times D_{m}$ and $\{m\} \times D_{m}^{\prime}$ converge to $x_{m} \in X$.

3.3. It is also seen that all compactifications of $\Delta^{\prime}$ contain $\beta N$. In order to obtain a first-countable, Lindelöf, Čech-complete space $X$ without maximal extension, we 
employ Alexandroff-Urysohn's two arrow space [E, Exercise 3.10.C]. That is, define

$$
X=(0,1] \times\{0\} \cup[0,1) \times\{1\} \cup Q \times N,
$$

where $Q$ denotes the set of rational numbers in $[0,1]$. All points of $Q \times N$ are defined to be isolated. To define neighborhoods at other points we need some notation. For $t \in[0,1]$, let $\lambda^{+}(t)$ and $\lambda^{-}(t)$ be strictly increasing and decreasing sequences, respectively, of rational numbers which converge to $t$. We assume that $\lambda^{+}(0)=\lambda^{-}(1)=\varnothing$. Let $\{A(x) \mid x \in(0,1] \times\{0\} \cup[0,1) \times\{1\}\}$ be the collection of all subsets of $N$. The neighborhood basis at $(t, 0) \in(0,1] \times\{0\}$ is defined as $U_{n}$, $n=1,2, \ldots$, where $U_{n}$ is the union of $\{(t, 0)\},\{(s, i) \mid t-1 / n<s<t$ and $i=0,1\}$, $\left(\lambda^{-}(t)-\right.$ (finite set) $) \times A(t, 0)$ and $U\{\{q\} \times N \mid t-1 / n<q<t\}-\lambda^{+}(t) \times$ $A(t, 1)$. Neighborhood bases at points of $[0,1) \times\{1\}$ are defined likewise. The Čech-completeness and nonexistence of a maximal extension of $X$ could be shown in the same way as 3.2 .

\section{REFERENCES}

[A] A. V. Arhangel'skiì, Structure and classification of topological spaces and cardinal invariants, Uspehi Mat. Nauk 33 (1978), no. 6, 29-84 = Russian Math. Surveys 33 (1978), no. 6, 33-96.

[vDP] E. K. van Douwen and T. C. Przymuśinski, First-countable and countable spaces all compactifications of which contain $\beta N$, Fund. Math. 102 (1979), 229-234.

[E] R. Engelking, General topology, PWN, Warsaw, 1977.

[N] J. Nagata, Modern general topology, North-Holland, Amsterdam, 1968.

[S] R. M. Stephenson, Jr., Minimal first countable topologies, Trans. Amer. Math. Soc. 138 (1969), 115-127.

[T] J. Terasawa, Spaces $N \cup \mathcal{R}$ and their dimensions, Topology Appl. 11 (1980), 93-102.

[W] R. Walker, The Stone-Čech compactification, Springer-Verlag, Berlin, Heidelberg and New York, 1974.

Department of Mathematics, Faculty of Engineering, Yokohama National University, YOKOHAMA 240, JAPAN

Department of Mathematics, The Defence Academy, Yokosuka 239, Japan 